\title{
Where and when are portion sizes larger in young children? An analysis of eating occasion size among 1.5-5-year-olds in the UK National Diet and Nutrition Survey (2008-2017)
}

\author{
Alice Porter ${ }^{1,2, *}$ (), Zoi Toumpakari ${ }^{2}$ (1), Ruth Kipping ${ }^{1}$, Carolyn Summerbell ${ }^{3,4}$ and \\ Laura Johnson ${ }^{2}$ \\ 'Population Health Sciences, Bristol Medical School, University of Bristol, Bristol, UK: ${ }^{2}$ Centre for Exercise, Nutrition \\ and Health Sciences, School for Policy Studies, University of Bristol, Bristol BS8 2BN, UK: ${ }^{3}$ Department of Sport and \\ Exercise Sciences, Durham University, Durham, UK: ${ }^{4}$ Fuse, NIHR Centre for Translational Research in Public Health, \\ London, UK
}

Submitted 3 March 2021: Final revision received 20 December 2021: Accepted 22 December 2021: First published online 27 December 2021

\begin{abstract}
Objective: To identify eating occasion-level and individual-level factors associated with the consumption of larger portions in young children and estimate their relative importance.

Design: Cross-sectional.

Setting: Data from parent-reported 4-d food diaries in the UK National Diet and Nutrition Survey (2008-2017) were analysed. Multilevel models explored variation in eating occasion size (kJ) within ( $n 48419$ occasions) and between children ( $n$ 1962) for all eating occasions. Eating contexts: location, eating companion, watching TV, and sitting at a table and individual characteristics: age, gender, ethnicity and parental socio-economic status were explored as potential correlates of eating occasion size.

Participants: Children aged $1 \cdot 5-5$ years.

Results: Median eating occasion size was 657 kJ (IQR 356, 1117). Eating occasion size variation was primarily attributed (90\%) to differences between eating occasions. Most (73\%) eating occasions were consumed at home. In adjusted models, eating occasions in eateries were $377 \mathrm{~kJ}$ larger than at home. Eating occasions sitting at a table, $v$. not, were $197 \mathrm{~kJ}$ larger. Eating in childcare, with additional family members and friends, and whilst watching TV were other eating contexts associated with slightly larger eating occasion sizes.

Conclusions: Eating contexts that vary from one eating occasion to another are more important than demographic characteristics that vary between children in explaining variation in consumed portion sizes in young children. Strategies to promote consumption of age-appropriate portion sizes in young children should be developed, especially in the home environment, in eating contexts such as sitting at the table, eating with others and watching TV.
\end{abstract}

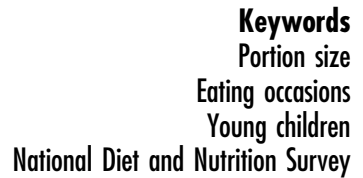

Keywords

Eating occasions

Young children

National Diet and Nutrition Survey
Childhood obesity is a worldwide public health problem, with 38 million children under the age of 5 years classified as overweight or obese in $2019^{(1)}$. Large portion sizes are suggested to contribute to childhood obesity ${ }^{(2)}$.

Experimental evidence has established a link between serving large portions and greater energy intake (EI) in young children, defined as the 'portion size effect'(3,4). The effect has been observed for meals and snacks, and across consecutive days ${ }^{(5)}$, however may vary depending on the individual, food or environment ${ }^{(6,7)}$.
To better understand this variability, we need to explore which factors are associated with the consumption of large portions in children. Several factors such as genetic susceptibility, responsiveness to food, parent feeding styles and the home food environment have been proposed to increase a child's behavioural susceptibility to consuming large portions ${ }^{(7)}$ and increase weight ${ }^{(8,9)}$. Existing research has focused on individual factors and less is known about within-person factors such as eating environments. 
Observational studies add to the experimental literature by exploring portion sizes in free-living settings and in larger, more diverse samples. The National Diet and Nutrition Survey (NDNS) is a nationally representative cross-sectional survey, which collects dietary data from children and adults in the $\mathrm{UK}^{(10)}$. Although data on served portions are not collected, the data provide estimates of portions consumed. These data can be used to explore potential factors associated with the intake of larger portions, a more proximal factor on the proposed causal pathway from larger servings to excessive consumption and subsequent weight gain.

Previous studies using NDNS data have observed associations with the consumption of individual foods, in children and adolescents ${ }^{(11-14)}$. Consuming larger portions (g) of energy-dense foods such as chocolate, confectionary, savoury snacks and biscuits was associated with eating out of the home and watching TV, being older, male and having lower household income. Eating out of home and with friends was also associated with greater non-core EI (kcal) (e.g soft drinks, savoury snacks and chocolate) in adolescents ${ }^{(13)}$. Larger consumed portions of vegetables $(\mathrm{g})$ were observed during the weekend and the evening meal, whilst eating at home and among older children ${ }^{(12)}$. Not watching TV and sitting at a table were also associated with greater vegetable consumption $(\mathrm{g})^{(14)}$. These studies provide insight into which eating contexts and individual characteristics may lead to the consumption of larger portion of individual foods. Consuming larger portions of low energy-dense foods, such as fruit and vegetables, can be beneficial for children's health ${ }^{(15)}$. In a meal, increasing the portion of fruit or vegetables will increase the volume $(\mathrm{g})$ of the portion but, owing to their low energy density, may decrease the total energy consumed $(\mathrm{kJ} / \mathrm{kcal})$ from that meal ${ }^{(16)}$. Overall meal size (kcal) (regardless of food type) has been prospectively associated with excessive weight gain in young children ${ }^{(17)}$. Therefore, it is important to explore associations in relation to the overall energy content of eating occasions (referred to hereafter as eating occasion size), where foods and beverages are consumed in combination.

We also need to understand the relative importance of eating environments $v$. individual characteristics. In previous studies, $89 \%$ of variability in non-core food intake $^{(13)}$ and $82 \%$ of variability in consumed vegetable portions $^{(12)}$ were attributed to differences between eating occasions. This suggests targeting high-risk environments could be more effective if prioritised over specific person-level characteristics. Understanding whether variability in eating occasion size is attributable to differences between eating occasions or between young children, and the eating contexts and individual characteristics associated with larger eating occasions could help us to understand when, where and for who the risk of consuming larger portions is higher.
Young children eat in distinct environments, with typically less control over their food choices than older children, and may have increased susceptibility to the portion size effect ${ }^{(18)}$. Although many portion size guidance resources aimed at feeding young children (referred to as 1 to 5 years of age) are available in the UK, many are not informed by the portion size research ${ }^{(19)}$. Therefore, exploring factors associated with portion size could help contribute to the call for improvement of nutrition guidelines that are research-driven, contextually specific and based on causal mechanisms ${ }^{(20)}$.

This study aimed to describe young children's eating occasions and to explore the relative contributions of within-children (between eating occasions) and betweenchildren variation in eating occasion size. We aimed to identify possible eating contexts and individual characteristics associated with larger eating occasion size $(\mathrm{kJ})$ in young children (aged 1 to 5 years).

\section{Methods}

\section{Study sample}

Secondary data analysis was conducted on dietary data from 1962 young children aged 1.5-5 years in the UK NDNS Years 1 to $9(2008 / 2009-2017)$ rolling programme. The survey design has been described elsewhere ${ }^{(21)}$. NDNS data were downloaded from the UK Data Archive $^{(22)}$.

\section{Dietary data}

Dietary data were collected via 4-d estimated food diaries, completed by parents of the participating children. Parents were asked to record all foods and beverages consumed, including the day and exact time. Parents estimated portion sizes using household measures (e.g. tablespoons), grams from packaging and example pictures provided ${ }^{(23)}$. Diaries were coded by a trained NDNS research team. Where grams were not reported, portion sizes were determined by coders using household measures in the Diet In Nutrients Out system ${ }^{(24)}$ or available packaging. Portion sizes were converted into energy by the NDNS research team using the food composition data from the Department of Health NDNS nutrient databank.

\section{Definition of eating occasions}

The outcome of interest was eating occasion size, measured in kilojoules $(\mathrm{kJ})$. Eating occasions were defined as an occasion in which energy-containing foods or beverages were consumed within the same 15 -min period, as defined in previous eating patterns research in children $^{(11,25-27)}$. If two or more items were consumed within $15 \mathrm{~min}$, these were considered a single eating occasion, if $>15$ min separated reported items, these were considered separate eating occasions. 


\section{Eating occasion variables}

Parents of participants completed a face-to-face computerassisted personal interview and questionnaires. Parents were asked to record where and with whom (eating companion) each food and beverage was consumed. The original 'where' and 'with whom' variables were recoded into six and five categories, respectively, similar to previous research ${ }^{(28)}$ (see online supplementary material, Supplemental Table S1 and Table S2). Parents were asked to record whether each food and beverage was eaten sat at the table or watching TV. Where watching TV responses were not specified, we classified these as 'not watching TV' (17\% of occasions).

\section{Individual variables}

Individual characteristics such as child's gender, age (years), ethnicity and total daily EI were available in the NDNS data. Height and weight data were measured by the interviewer and used to derive BMI z-scores using the BMI WHO cut-offs for 2-3-year-olds ${ }^{(29)}$ and UK90 for 4-5-year-olds ${ }^{(30,31)}$. Parental socio-economic status (SES) was indicated by parental occupation using the National Statistics Socio-Economic Classification (NSSEC) ${ }^{(32)}$ (see online supplementary material, Supplemental Table S3). Misreporting of EI was assessed using the individualised method for children ${ }^{(33,34)}$, which involved calculating the ratio of reported EI to estimated energy requirements, accounting for growth. Plausible reporting of EI was identified using cut-offs of 0.79 and 1.21 . Seventeen per cent of the total sample were categorised as under-reporters and $20 \%$ as over-reporters.

\section{Statistical analysis}

\section{Descriptive analysis}

All analyses were conducted in Stata 15. Exposure variables included four eating occasion characteristics (eating contexts): location, eating companion, watching TV, and sitting at a table, and four individual characteristics: age, gender, ethnicity, and parental SES. Descriptive statistics on characteristics of eating occasions were reported at the survey level (across all young children). Number and frequency of eating occasions and median (and interquartile range (IQR)) eating occasion size were reported for each eating context variable. Descriptive statistics on individual characteristics were reported. Number (\%) of children was reported for categorical variables and mean and standard deviation for continuous variables. Mean eating occasion frequency and median eating occasion size were reported for categorical variables. For continuous variables, simple regression analyses were conducted, and $\beta$-coefficients (B) and $95 \%$ CI were reported. The number of young children who reported to consume an eating occasion in each of the eating contexts was presented across all children and by individual characteristics, to understand how these variables were inter-related.

Energy density of eating occasions (as defined above) was calculated (kilojoules of eating occasion divided by grams of eating occasion) and median (IQR) was reported. Simple analysis of food groups associated with larger eating occasions was conducted. The food groups classified within the NDNS were collapsed further according to the UK Eatwell Guide ${ }^{(35)}$ food groups (starchy, protein, fruit and vegetables, dairy, oils and spreads, foods high in fat and sugar, and drinks) ${ }^{(19)}$. The percentage of all eating occasions in which young children consumed a given food group was reported. Spearman's correlations were conducted to explore correlations between percentage of total energy consumed in an eating occasion from a food group and overall eating occasion size.

\section{Multilevel modelling}

Hierarchical multilevel modelling ${ }^{(36)}$ was used to explore the relationship of eating occasion size with eating contexts and individual characteristics as potential exposure variables. Eating occasions (level 1 variation) are nested within children (level 2 variation). Therefore, multilevel modelling allowed us to explore whether eating occasion size varied within and between children, as well as the potential exposures that explained this variability. Eating occasion size $(\mathrm{kJ})$ was not normally distributed and was logged transformed to approximate the normal distribution. Individual-level survey weights from each survey wave were combined according to NDNS instructions $^{(10)}$ and used in analyses to account for selection and non-response biases.

Several models were run: Model 1 was the variance component (null intercept) model, which did not include any exposure variables. This model assessed how much variability in eating occasion size was attributable to within-children-between-eating occasions and betweenchildren variance. Models 1.1 to 1.8 explored the unadjusted associations between each of the eight exposures of interest and eating occasion size in their own model. In Models $2 \cdot 1$ to $2 \cdot 8$, a set of confounders unique to each of the eight exposures of interest were added to each model to explore if the evidence and size of associations were robust to adjustment for potential confounding. Supplemental Table S4 provides a description of each model, including the potential confounders added for each exposure at each stage. Models $2 \cdot 1$ to $2 \cdot 8$ were adjusted for misreporting of EI as a potential confounder, because misreporting has previously been shown to affect diet-health relationships ${ }^{(37-39)}$. Individual-level models $(2 \cdot 5-2 \cdot 8)$ were adjusted for total daily $\mathrm{EI}^{(40)}$.

For each model, the intraclass correlation and 'percentage variance explained' were calculated. These indicated the percentage of variation in eating occasion size attributed to differences at our two levels of variation and how much variance could be explained by our exposure 
variables compared to the null-intercept model, respectively. Model fit was assessed using likelihood ratio tests. Estimates were converted to kilojoules ( $\mathrm{kJ}$ ) by multiplying the adjusted ratios by the model intercept, to provide meaningful public health units.

The STROBE flowchart ${ }^{(41)}$ (see online supplementary material, Supplemental Fig. S1) illustrates the amount of missing data in the sample. We reported the sample size of each model and used the likelihood ratio test to assess whether missing data could bias our results.

\section{Mediation analyses}

To aid interpretation of the results from Models $2 \cdot 1$ to $2 \cdot 8$, mediation analysis was conducted. Eating occasion type (whether an eating occasion was defined as a meal or snack) and eating frequency (average number of daily eating occasions) were added as potential mediators to the eating occasion-level and child-level models, respectively, to explore whether potential associations observed were due to children consuming specific eating occasion types or eating more frequently. Each eating occasion was defined as a meal or snack using a time of day, plus energy criterion method based on our data, similar to previous research $^{(42-44)}$. The percentage energy from each eating occasion (of total daily EI) was plotted in 30-min intervals over a 24-h period, across all participants. The resulting graph (see online supplementary material, Supplemental Fig. S2) displayed three peaks in energy across the day, which were used to label eating occasions as meals or snacks. We defined meals as eating occasions with the largest percent energy between $05.30-10.00,11.00-14.00$ and 16.00-19.00. All other smaller eating occasions within these mealtimes and all eating occasions outside of these mealtimes were defined as snacks. In the descriptive results, eating occasion size and frequency were additionally reported for meals and snacks because meals and snacks are systematically different in size. In the multilevel models, potential mediators were added to Models $2 \cdot 1$ to $2 \cdot 8$ if an exposureoutcome association was observed. Estimates from the mediation models were compared to the final adjusted estimates to explore potential mediation. Models 3.1 to 3.8 present the mediation models and are presented as the final models because including eating occasion type and eating frequency provided the most meaningful interpretation of results within the context of the study.

\section{Results}

\section{Descriptive results}

Characteristics of eating occasions

The median eating occasion size across all young children ( $n$ 1962) and all eating occasions ( $n 48$ 219) was $657 \mathrm{~kJ}$ (IQR 356, 1117). The median size for meals was $1050 \mathrm{~kJ}$ (IQR 711, 1506) and for snacks was $402 \mathrm{~kJ}$ (IQR 209,
640). On average, young children consumed 6.7 (SD 1.8) eating occasions per $\mathrm{d}$, of which 3.0 (SD 0.3) were meals and 3.7 (SD 1.9) were snacks.

Supplemental Table S5 displays the number (\%) of eating occasions across the different eating contexts. Nearly three-quarters of eating occasions occurred at home, with $11 \%$ in childcare and just $2 \%$ in eateries. Meals made up $47 \%$ and snacks $53 \%$ of eating occasions overall, whereas $63 \%$ of occasions in eateries were meals and $77 \%$ of eating 'on the go' was a snack. Parents and/or other family members ate with young children in $85 \%$ of eating occasions, with just $5 \%$ eaten alone. A third of occasions were while watching TV and nearly half were while sitting at a table. Supplemental Figure S3 illustrates the median (IQR) eating occasion, meal and snack sizes across the different eating contexts.

Supplemental Table 56 suggests larger eating occasions were more energy-dense than smaller eating occasions $(4.6 \mathrm{~kJ} / \mathrm{g} v .1 .7 \mathrm{~kJ} / \mathrm{g})$ and contained more food groups (the percentage of all eating occasions in which young children consumed a given food group was greater across all food groups for larger $v$. smaller eating occasions). Supplemental Table S7 suggests percentage energy from all food groups (but not drinks) were correlated with overall eating occasion size.

\section{Characteristics of young children}

Table 1 describes the sample of young children ( $n$ 1962). The sample consisted of $53 \%$ boys, $86 \%$ White ethnicity, $39 \%$ low SES, with a mean child age of 3 years (SD 1.3). Supplemental Figure 44 presents the median (IQR) eating occasion, meal and snack sizes across the individual characteristics. The overall frequency of eating occasions was similar among boys and girls, and SES groups but varied by ethnicity; 7.1 times/d among Asian/Asian British children $v .5 .6$ times/d among Black/Black British children. A lower eating occasion frequency and greater eating occasion size was associated with being older $(0.3$ eating occasions less per $\mathrm{d}$ and $92 \mathrm{~kJ}$ more per occasion, per year of age). A higher eating frequency was associated with smaller eating occasions ( $-67 \mathrm{~kJ}$ per occasion for each extra time eating occurred). A higher total EI was associated with larger eating occasions ( $52 \mathrm{~kJ}$ per occasion for each $418 \mathrm{~kJ}$ of total energy consumed) (data not shown, table available upon request).

Characteristics of young children within eating contexts All young children reported eating at home, $60 \%$ in childcare and $51 \%$ 'on the go'. Fewer young children ate at a friend's or relative's house ( $46 \%$ ), in eateries (34\%) and at activity places ( $40 \%$ ). Eighty per cent of children ate with their parents/carers and $77 \%$ with family and friends. Fewer children ate with parents and siblings (54\%) and with friends (54\%). Only $36 \%$ of children ate alone. Most children reported eating watching TV (92\%) and not watching TV (99\%). Similarly, $96 \%$ of children reported 
Table 1 Characteristics of young children $1-5$ years $(n 1962)$ in the UK National Diet and Nutrition Survey 2008-2017

\begin{tabular}{|c|c|c|c|}
\hline & $n$ & $\%$ & \\
\hline \multicolumn{4}{|l|}{ Gender } \\
\hline Boys & 1034 & 53 & \\
\hline Girls & 928 & 47 & \\
\hline \multicolumn{4}{|l|}{ Socio-economic status } \\
\hline Low & 744 & 39 & \\
\hline Intermediate & 368 & 19 & \\
\hline High & 812 & 42 & \\
\hline \multicolumn{4}{|l|}{ Ethnicity } \\
\hline White & 1688 & 86 & \\
\hline Black/Black British & 49 & 3 & \\
\hline Asian/Asian British & 122 & 6 & \\
\hline Mixed & 64 & 3 & \\
\hline Other & 39 & 2 & \\
\hline \multicolumn{4}{|l|}{ Misreporting of energy intake } \\
\hline Plausible reporter & 1233 & 63 & \\
\hline Under-reporter & 338 & 17 & \\
\hline \multirow[t]{2}{*}{ Over-reporter } & 391 & 20 & \\
\hline & $n$ & Mean & SD \\
\hline Age (years) & 1962 & $3 \cdot 1$ & $1 \cdot 3$ \\
\hline BMl z-score* & 1593 & 0.4 & $1 \cdot 2$ \\
\hline Total daily eating frequency & 1962 & $6 \cdot 7$ & $1 . \overline{8}$ \\
\hline Total daily energy intake (kJ) & 1962 & 5012 & 1163 \\
\hline
\end{tabular}

${ }^{*}$ Calculated using WHO BMI z-scores for $1.5-3$ years and UK 1990 BMI z-score $4-5$ years.

eating sitting at a table and $92 \%$ whilst not. Sitting at a table $v$. not was more common in childcare $(68 \%)$ and eateries (74\%); more common between 12.00 and 14.00 (61\%) and less common after 20:00 (17\%); more common when eating with friends (67\%) and less common when eating alone (24\%) (data not shown, table available upon request)

\section{Multilevel model results}

Associations of eating contexts with eating occasion size Figure 1 presents the association of eating contexts with eating occasion size in kilojoules from Models 3.1 to 3.4 (adjusted for potential confounders and mediators). Table 2 presents the ratios and $95 \%$ CI from Models $3 \cdot 1$ to $3 \cdot 4$. Model 3 provided the best model fit (see online supplementary material, Supplemental Table S9) and allowed for the most meaningful interpretation of results. Supplemental Table S8 presents the ratios and 95\% CI from Models 1.1 to 1.4 and 2.1 to 2.4 before adjustment for potential mediators. Eating in eateries was associated with the largest eating occasion size in young children, being over $50 \%$ larger than eating at home, equating to a difference of $377 \mathrm{~kJ}$. Eating sitting at a table was associated with a larger eating occasion size; $197 \mathrm{~kJ}$ larger $v$. not sitting at a table. Eating in childcare and at a friend's or relative's house were associated with larger eating occasion sizes, compared to eating at home (121 kJ and $63 \mathrm{~kJ}$ larger, respectively). Eating with parents and siblings, and family and friends were associated with slightly larger eating occasion sizes, equating to $59 \mathrm{~kJ}$ and $71 \mathrm{~kJ}$ larger than eating with parents only, respectively. Eating alone was associated with smaller eating occasion size; $113 \mathrm{~kJ}$ smaller than eating with parents. Eating occasions were slightly larger when watching TV $v$. not, equating to a $46 \mathrm{~kJ}$ difference. Eating occasion type was added as a potential mediator to the models to account for meals potentially being more frequently consumed in certain eating contexts than snacks and therefore explaining why eating occasion size is larger (because meals are systematically larger than snacks). After adding eating occasion type, eating on-the-go, at activity places and with friends were no longer associated with eating occasion size (fully mediated relationship). Estimates were partially mediated after adding eating occasion type for the sitting at a table and eating companion variables (Table 2 , see online supplementary material, Supplemental Table S8).

\section{Associations of individual characteristics with eating occasion size}

Figure 2 presents the association of individual characteristics with eating occasion size in kilojoules from Models 3.5 to 3.8 . Table 2 presents the ratios and $95 \%$ CI from Models 3.5 to 3.8. Young children of Black, Asian, Mixed and Other ethnicities had eating occasion sizes slightly larger than children of White ethnicity, by 96 kJ, 92 kJ, 79 kJ and 113 $\mathrm{kJ}$, respectively. Gender and parental SES showed no evidence of association with eating occasion size. Eating frequency was added as a potential mediator to the models to account for eating occasion size being larger due to eating less frequently. After adding eating frequency, being older was no longer associated with eating occasion size (fully mediated relationship) (Table 2 , see online supplementary material, Supplemental Table S8).

\section{Explaining eating occasion size variation by eating occasion $v$. child characteristics}

Supplemental Table S9 presents the variance estimates for each of the multilevel models. The null-intercept model showed most of the variation in eating occasion size was attributed to characteristics of the eating occasion (90\% variance), leaving just $10 \%$ variation attributable to characteristics of the young children. Eating contexts (location, eating companion, watching TV and sitting at a table) explained $16 \%$ of the total variance in eating occasion size, whereas the individual characteristics (age, gender, ethnicity and parental SES) explained just $2 \%$. When all exposures and confounders were added, total variance explained was $23 \%$, which increased to $41 \%$ when mediators (eating occasion type and eating frequency) were added.

\section{Discussion}

Ninety per cent of the variation in eating occasion size was within children, with only $10 \%$ attributed to differences between children. Eating contexts explained $16 \%$ of the total variance in eating occasion size, compared to only 
(a)

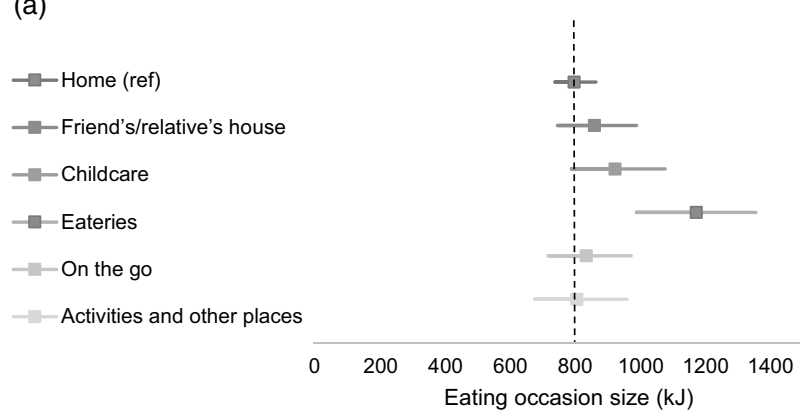

(c)

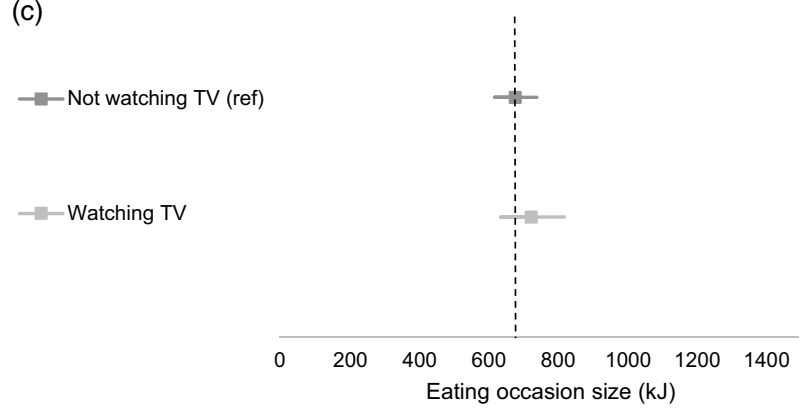

(b)

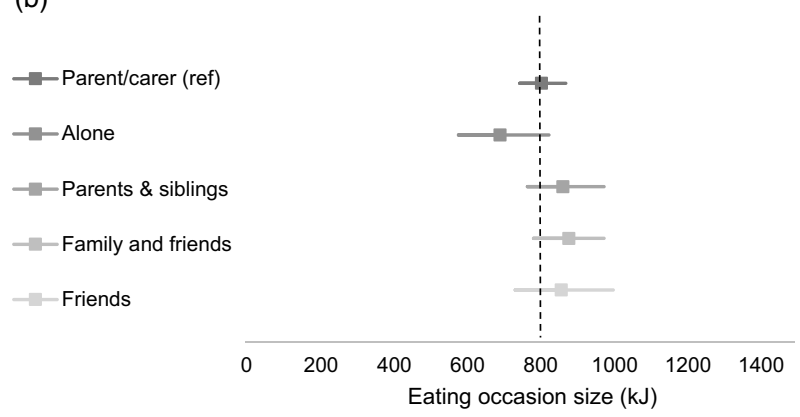

(d)

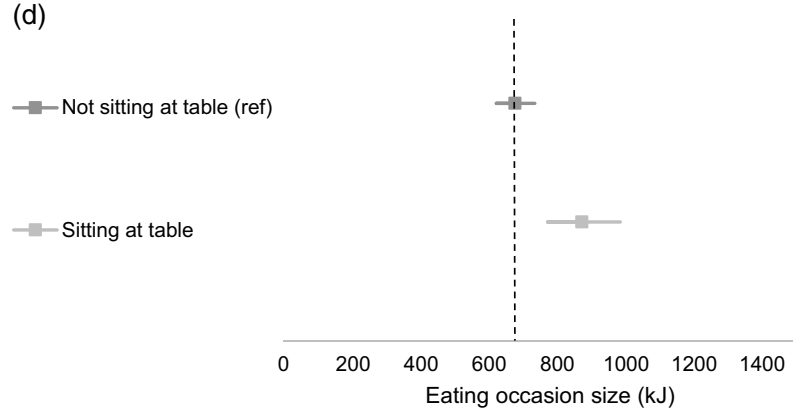

Fig. 1 Associations of eating contexts with eating occasion size among young children 1-5 years, in the National Diet and Nutrition Survey 2008-2017. *Computed from Supplemental Table S8 (estimate $=$ intercept $\times$ ratio). Estimate shows the eating occasion size $(\mathrm{kJ})$ that young children reported for each eating context. $95 \% \mathrm{Cl}$ are represented by the horizontal lines. Vertical dashed lines represent the eating occasion size for each reference category (intercept) adjusted for confounders and allows comparison of eating occasion size with other categories

$2 \%$ explained by individual characteristics. These findings suggest factors that differ from one occasion to another (such as eating contexts) can better help us to understand why portion sizes are larger in this sample of young children than factors that differ from one child to another (such as individual characteristics). Our findings align with Toumpakari et al. ${ }^{(13)}$ who found $89 \%$ of variation in noncore EI in adolescents was attributed to characteristics of the eating occasions. We therefore support future research and guidance to focus on the eating environment in young children $^{(19,45)}$.

Public Health England (PHE) recommended children should only consume two $100 \mathrm{kcal}$ ( $418 \mathrm{~kJ}$ ) snacks (excluding fruit and vegetables) per $\mathrm{d}^{(46)}$. Our findings suggest young children in this sample, on average consumed more than three $402 \mathrm{~kJ}$ snacks per d, which could exceed PHE recommendations. Median meal size was $1050 \mathrm{~kJ}$. A systematic review of resources recommending portion sizes for 1-5-year-olds found recommended meal sizes across resources were between $473 \mathrm{~kJ}$ and $1761 \mathrm{~kJ}^{(19)}$. A comparison should be interpreted with caution because several resources included in the review recommended portion sizes to meet energy requirements of 3-5-year-olds (whereas this sample also included younger children, with lower energy requirements). If following certain recommendations, such as those from the Infant and Toddler Forum $^{(47)}$, young children in this sample could be consuming larger meals than recommended. This highlights the need to promote consumption of age-appropriate portion sizes to meet energy requirements.

Sitting at a table was independently associated with an eating occasion size on average $197 \mathrm{~kJ}$ larger than not sitting at a table. Compared to eating with parents only, eating occasion size was larger when eating with parents and siblings and with family and friends, by $59 \mathrm{~kJ}$ and $71 \mathrm{~kJ}$, respectively. Compared to eating at home, eating occasion size was larger when eating in childcare and at a friend's or relative's house, by $121 \mathrm{~kJ}$ and $63 \mathrm{~kJ}$, respectively. Although portion sizes may be larger in these contexts, this is only problematic if portions are large enough to result in surplus $\mathrm{EI}$, as this could lead to excessive weight gain ${ }^{(17)}$. It may be larger portions of healthy foods, such as vegetables are being consumed in these contexts ${ }^{(14)}$, due to larger servings ${ }^{(48)}$, or consumption being encouraged and modelled by others ${ }^{(49)}$. However, large portions of vegetables are not likely to result in energy-dense eating occasions because of their low-energy content. If high energy-dense foods such as desserts are being consumed in these contexts $^{(50)}$, this could substantially increase the energy content of an eating occasion and lead to a surplus EI. Our results suggest larger eating occasions were more energy-dense, contained more food groups and were being driven the most by a greater percentage energy from starchy foods and proteins (see online supplementary material, Supplemental Table S6 and S7). The findings suggest parents and childcare settings may need 
Table 2 Relationship of eating occasion size with eating contexts and individual characteristics among young children $1-5$ years $(n 1962)$ in the UK National Diet and Nutrition Survey 2008-2019. Presents results from Model 3

\begin{tabular}{|c|c|c|c|}
\hline \multirow[b]{2}{*}{ Exposure } & \multicolumn{3}{|c|}{$\begin{array}{c}\text { Models } 3 \cdot 1-3 \cdot 8-\text { Adjusted for potential confounders and mediators } \\
\text { (eating occasion type and eating frequency) }\end{array}$} \\
\hline & Ratio* & $95 \% \mathrm{Cl}$ & $P$-value \\
\hline \multicolumn{4}{|l|}{ Eating contexts } \\
\hline \multicolumn{4}{|l|}{ Model 3.1 - location† } \\
\hline Home (ref) & 1.00 & & \\
\hline Friend's/relative's house & 1.08 & $1.02,1.14$ & 0.014 \\
\hline Childcare & 1.15 & $1.07,1.24$ & $<0.001$ \\
\hline Eateries & 1.47 & $1.34,1.56$ & $<0.001$ \\
\hline On the go & 1.05 & $0.98,1.13$ & 0.199 \\
\hline Activities and other places & 1.01 & $0.92,1.11$ & 0.857 \\
\hline \multicolumn{4}{|l|}{ Model $3 \cdot 2$ - eating companion $\ddagger$} \\
\hline Parent/carer (ref) & 1.00 & & \\
\hline Alone & 0.86 & $0.78,0.95$ & 0.002 \\
\hline Parents and siblings & 1.07 & $1.03,1.12$ & 0.002 \\
\hline Family and friends & 1.09 & $1.05,1.13$ & $<0.001$ \\
\hline Friends & 1.06 & $0.99,1.15$ & 0.114 \\
\hline \multicolumn{4}{|c|}{ Model 3.3 - watching TV whilst eating§ } \\
\hline Not Watching TV (ref) & 1.00 & & \\
\hline Watching TV & 1.07 & $1 \cdot 03,1 \cdot 11$ & 0.001 \\
\hline \multicolumn{4}{|c|}{ Model $3.4-$ sitting at table whilst eatingll } \\
\hline Not sitting at table (ref) & 1.00 & & \\
\hline Sitting at table & 1.29 & $1 \cdot 24,1 \cdot 34$ & $<0.001$ \\
\hline \multicolumn{4}{|l|}{ Individual characteristics } \\
\hline \multicolumn{4}{|l|}{ Model 3.5 - age } \\
\hline 1 year (ref) & 1.00 & & \\
\hline 2 years & 1.04 & $0.94,1.14$ & 0.479 \\
\hline 3 years & 0.97 & $0.87,1.07$ & 0.541 \\
\hline 4 years & 1.00 & $0.89,1.13$ & 0.947 \\
\hline 5 years & 1.00 & $0.89,1.12$ & 0.995 \\
\hline \multicolumn{4}{|l|}{ Model 3.6 - gender } \\
\hline Boys (ref) & 1.00 & & \\
\hline Girls & 0.99 & $0.95,1.02$ & 0.407 \\
\hline \multicolumn{4}{|l|}{ Model 3.7 - ethnicity } \\
\hline White (ref) & 1.00 & & \\
\hline Black/Black British & 1.20 & $1 \cdot 12,1 \cdot 27$ & $<0.001$ \\
\hline Asian/Asian British & $1 \cdot 19$ & $1 \cdot 12,1.26$ & $<0.001$ \\
\hline Mixed & $1 \cdot 16$ & $1.09,1.23$ & $<0.001$ \\
\hline Other & 1.23 & $1.14,1.32$ & $<0.001$ \\
\hline \multicolumn{4}{|l|}{ Model 3.8 - parental SES ${ }^{* *}$} \\
\hline Low (ref) & 1.00 & & \\
\hline Intermediate SES & 1.02 & $0.96,1.08$ & 0.518 \\
\hline High SES & 1.04 & $0.99,1.08$ & 0.118 \\
\hline
\end{tabular}

SES, socio-economic status.

*To improve interpretability, ratios are presented as the exponentiated values of the log-transformed coefficients and represent changes in the ratio of the mean eating occasion size. For example, an exponentiated value of 1.14 represents a $14 \%$ difference in eating occasion size between the specified eating context/individual characteristic and its reference category.

†Adjusted for time of day, day of week, day number, age, ethnicity, parental SES and misreporting (and eating occasion type as potential mediator).

†Adjusted for time of day, day of week, location, day number, age, ethnicity and misreporting (and eating occasion type as potential mediator).

§Adjusted for time of day, day of week, location, eating companion, sitting at the table, day number, age, ethnicity, parental SES and misreporting (and eating occasion type as potential mediator).

IIAdjusted for time of day, day of week, location, eating companion, watching TV, day number, age, ethnicity and misreporting (and eating occasion type as potential mediator). ๆAdjusted for misreporting, total daily energy intake and zBMI (and eating frequency as potential mediator).

${ }^{\star \star}$ Adjusted for misreporting, total daily energy intake, ethnicity and zBMI (and eating frequency as potential mediator).

children eat. Certain practices and behaviours, such as encouraging plate cleaning, can lead parents to override their children's ability to self-regulate their intake, leading to long-term over-consumption ${ }^{(53)}$. It may be that when children eat at the table with their parents (and others), the social influences contribute towards consuming more. Interventions targeting parent feeding styles ${ }^{(55,56)}$ should incorporate portion size advice to help promote children's self-regulation from a young age. 
(a)

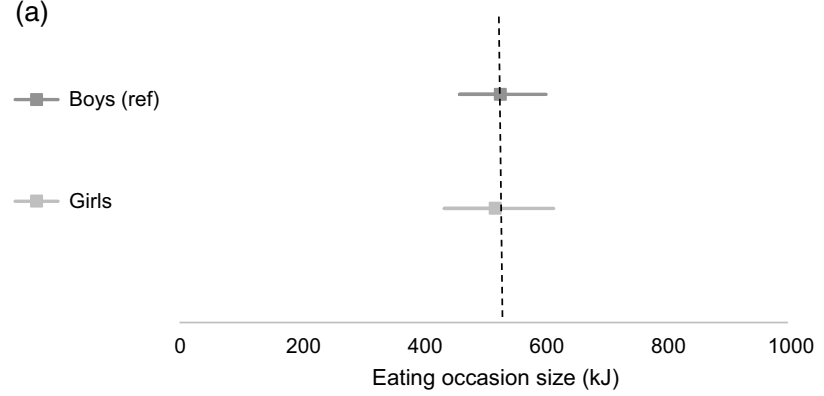

(c)

$$
\begin{aligned}
& --1 \text { year (ref) } \\
& --2 \text { years } \\
& --3 \text { years } \\
& -\square-4 \text { years } \\
& -\square-5 \text { years }
\end{aligned}
$$
occasion size with other categories (b)

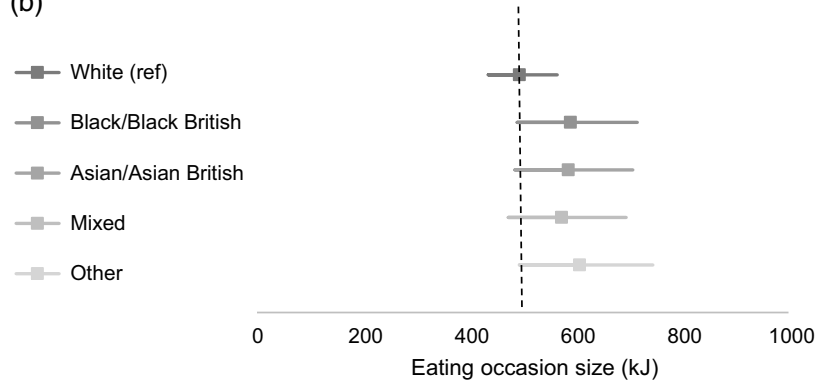

(d)

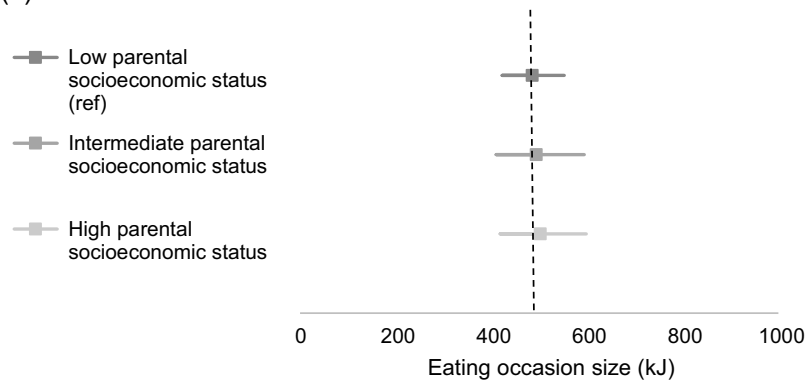

Fig. 2 Associations of individual characteristics with eating occasion size among young children 1-5 years, in the National Diet and Nutrition Survey 2008-2017. *Computed from Table S8 (estimate = intercept $\times$ ratio). Estimate shows the eating occasion size $(\mathrm{kJ})$ that young children report for each individual characteristic. $95 \% \mathrm{Cl}$ are represented by the horizontal lines. Vertical dashed lines represent the eating occasion size for each reference category (intercept) adjusted for confounders and allows comparison of eating

Eating in eateries (such as cafes, fast-food outlets and restaurants) was independently associated with the highest eating occasion size; on average, $377 \mathrm{~kJ}$ larger than eating at home. This is not surprising considering the existing literature suggesting restaurant meals (including children's meals) are large in portion size, too energydense $^{(51)}$ and do not meet nutritional standards ${ }^{(57)}$. In addition, eateries are associated with higher consumption of ultra-processed ${ }^{(58)}$ and non-core ${ }^{(13)}$ food, in children and adolescents. In our sample, only $34 \%$ of young children ate at eateries (only $2 \%$ of the total number of eating occasions). Similarly, Mak et al. ${ }^{(14)}$ found only $2.3 \%$ of the total eating occasions were consumed in eateries among 7-10-year-olds. However, large portion sizes served in eateries could influence consumption norms, by distorting both parents' and children's understanding of appropriate portion sizes ${ }^{(59)}$, especially when children are more susceptible to consuming large portions $^{(7)}$. As stated in the UK Childhood Obesity plan ${ }^{(60)}$, reducing energy content (and therefore portion size) of meals served in eateries could be a target for action. The number of out-of-home eating occasions may have been under-reported by parents due to the increased burden ${ }^{(61)}$. However, given the small number of eating occasions that were reported in eateries, to have a greater effect on reducing child population-level portion sizes, it may be more appropriate to target the home and childcare environments, where we found more meals and snacks were consumed ( $73 \%$ and $11 \%$, respectively).
Although we and others ${ }^{(11,62)}$ have identified individual characteristics and eating contexts associated with consuming larger portions, our models only explained $41 \%$ of the total variation. This suggests there are several other factors that need to be identified to fully explain why portion size varies in young children. A child's susceptibility to consume large portion sizes is due to a complex combination of nature (e.g. genetics), nurture (e.g. parent feeding practices), individual traits (e.g. satiety) and the environment (e.g. home food environment) ${ }^{(7)}$. Child-related factors such as eating traits and liking of the food, caregiver-related factors such as caregiver portion sizes and feeding practices ${ }^{(63)}$, and foodrelated factors such as energy density ${ }^{(64)}$ may all interact to influence the portion sizes children consume. Our results suggest we should focus on building the evidence base for factors associated with portion size that vary from one eating occasion to another (because this is where most of the variation in eating occasion size lies). Factors such as the food environment, child temperament, hunger and liking, parental feeding practices, serving method and food type should be further explored, whilst also considering how individual traits and characteristics may be bidirectionally related ${ }^{(7)}$.

Experimental portion size manipulation studies have shown significant increases in EI from a meal or snack, as a result of serving large portion sizes, by between 63 and $347 \mathrm{~kJ}^{(4,5,64-69)}$. Although we do not have data on the served portions and the data on consumed portions is an estimate of EI, we observed associations, which equated to eating occasions being between 46 and $377 \mathrm{~kJ}$ larger than 
reference category eating occasions. The difference in kilojoules is relatively small when comparing one eating context or child to another. However, if young children consistently consume meals or snacks in certain contexts or because of individual characteristics, which are associated with larger portions, this may have implications for excessive EI over time, and excessive weight gain ${ }^{(17,70)}$. Hebestreit et al. ${ }^{(71)}$ found daily food intake (g) and total EI (kcal) were positively associated with BMI z-score in 2-9-year-old children. However, in a combined model, only total EI was independently associated. As portion size is highly related to EI, the consistent consumption of large energy-dense portion sizes may contribute to excessive total EI and weight gain over time ${ }^{(17)}$. Therefore, the focus on age-appropriate portion sizes and $\mathrm{EI}$ is critical ${ }^{(72)}$. In addition, caregivers may benefit from guidance highlighting how the food environment can encourage the consumption of larger portions.

\section{Strengths and limitations}

Using multilevel modelling, we have accounted for the clustered hierarchical nature of our data (whereby eating occasions are nested within individuals). The multilevel models have accounted for both within and between variation in eating occasion size in young children, which minimises the potential biases related to person-level unmeasured variables associated with our outcome variable ${ }^{(58)}$. We used combined data from the NDNS Years 1-9, which enabled analysis of a large, UK nationally representative sample. We considered each exposure variable as a separate model to ensure appropriate adjustment for confounders and mediators, and to increase reliability.

The main limitation was energy density of and types of foods in the eating occasions were not analysed in detail, which limits our interpretations. Although based on previous research $^{(42,73)}$ and preliminary work, our chosen definition of eating occasion type may have inaccurately classified some eating occasions as meals and snacks and affected estimates. Despite this, Model 3, which included eating occasion type and eating frequency as potential mediators, was presented as the final model. Model 2 was provided for comparison between models. Including eating occasion type in Model 3 provided a more meaningful interpretation of the estimates, because only meals were compared to meals and only snacks compared to snacks, which accounted for the systematic difference in size between meals and snacks. For example, eating on-thego was associated with a smaller eating occasion size in Model 2 but in Model 3, when accounting for snacks being the predominant eating occasion on-the-go, an association was no longer observed. Similarly, including eating frequency in Model 3 provided more meaningful interpretation, because the size of an eating occasion may depend on how frequently a child eats.
Due to the cross-sectional nature of the data, our findings do not provide evidence of causation. Our sample included mostly White British young children (86\%) and so the findings may be less generalisable to other ethnic groups. Although, misreporting of EI was calculated and added to models, the parent-reported dietary data were subject to misreporting and subject bias ${ }^{(74)}$. The variables we selected for analysis only explained $41 \%$ of the variation in eating occasion size, which limits our interpretations. The survey lacked data on appetite traits and parental feeding behaviours ${ }^{(7)}$, which may have improved the percentage variance explained and enhanced our interpretations.

\section{Future research and policy implications}

Future research should continue to focus on eating habits of children and how these may affect EI, dietary intake and weight gain. Future research should pull together data or create new datasets that include all the factors previously associated with portion size in children, to better understand which factors have the greatest influence on increasing children's susceptibility to consuming larger portions. Future research should explore how the portion sizes of specific food groups or individual foods are combined and how they contribute to large eating occasions. It is also important to establish an accepted consensus for classifying eating occasions as meals and snacks where participant-reported eating occasions are not available. Future research should compare consumed meal and snack sizes reported in national surveys with the recommendations, to establish whether young children are overconsuming.

Governments and food industries should work together to agree on policies to reduce out-of-home portion sizes of children's meals and snacks. This could be achieved through the combination of reducing dishware and packet sizes $^{(75,76)}$, introducing calorie caps on meals in eateries (similar to the UK Soft Drinks Industry Levy) ${ }^{(77)}$ and/or price incentives for selecting smaller portions ${ }^{(78)}$.

\section{Conclusion}

To conclude, the variability in eating occasion size in young children is better explained by differences between eating occasions rather than individuals. Efforts to reduce portion sizes in children should focus on eating contexts rather than targeting children with certain demographic characteristics. Eating in eateries, sitting at a table, in childcare, with other family members and friends, and watching TV were all eating contexts associated with larger eating occasions. Effective strategies to promote the consumption of ageappropriate portion sizes, especially in the home environment, should be developed. 


\section{Acknowledgements}

Acknowledgements: The authors would like to thank the participants of and research teams behind the National Diet and Nutrition Survey, the reviewers for helping to improve the manuscript, and the Centre of Multilevel Modelling at the University of Bristol for their online resources on multilevel modelling. Financial support: This study is funded by the National Institute for Health Research (NIHR) School for Public Health Research (Grant PDSPH-2015). The NIHR School for Public Health Research is a partnership between the Universities of Sheffield, Bristol, Cambridge, Imperial, University College London, The London School for Hygiene and Tropical Medicine (LSHTM), LiLaC - a collaboration between the Universities of Liverpool and Lancaster and The Centre for Translational Research in Public Health (Fuse) a collaboration between Newcastle, Durham, Northumbria, Sunderland and Teesside Universities. The views expressed are those of the author(s) and not necessarily those of the NIHR or the Department of Health and Social Care. Conflict of interest: There are no conflicts of interest. Authorship: A.P. contributed to the conception, design and interpretation, undertook the data analysis and wrote the manuscript. Z.T. contributed to the interpretation and manuscript revisions. R.K. contributed to the conception, design, interpretation and manuscript revisions. C.S. contributed to the interpretation and manuscript revisions. L.J. contributed to the conception, design, interpretation and manuscript revisions. All authors read and approved the final manuscript. Etbics of buman subject participation: This study was conducted according to the guidelines laid down in the Declaration of Helsinki, and all procedures involving research study participants were approved by the Oxfordshire A Research Committee. Written informed consent was obtained from all subjects/patients.

\section{Supplementary material}

For supplementary material/s referred to in this article, please visit https://doi.org/10.1017/S1368980021005024

\section{References}

1. World Health Organization (2020) Obesity and Overweight: World Health Organization. https://www.who.int/newsroom/fact-sheets/detail/obesity-and-overweight (accessed June 2021).

2. Birch LL, Savage JS \& Fisher JO (2015) Right sizing prevention. Food portion size effects on children's eating and weight. Appetite 88, 11-16

3. Rolls BJ, Engell D \& Birch LL (2000) Serving portion size influences 5-year-old but not 3-year-old children's food intakes. J Am Diet Assoc 100, 232-234.

4. Fisher JO, Liu Y, Birch LL et al. (2007) Effects of portion size and energy density on young children's intake at a meal. AmJ Clin Nutr 86, 174-179.
5. Smethers AD, Roe LS, Sanchez CE et al. (2019) Portion size has sustained effects over $5 \mathrm{~d}$ in preschool children: a randomized trial. Am J Clin Nutr 109, 1361-1372.

6. Zuraikat FM, Smethers AD \& Rolls BJ (2019) Potential moderators of the portion size effect. Physiol Behav 204, 191-198.

7. Kral TVE \& Hetherington MM (2015) Variability in children's eating response to portion size. A biobehavioral perspective. Appetite 88, 5-10.

8. Schrempft S, van Jaarsveld CHM, Fisher A et al. (2018) Variation in the heritability of child Body Mass Index by obesogenic home environment. JAMA Pediatr 172, 1153-1160.

9. Kininmonth AR, Smith AD, Llewellyn $\mathrm{CH}$ et al. (2021) The relationship between the home environment and child adiposity: a systematic review. Int J Behav Nutr Phys Act 18, 4.

10. MRC Elsie Widdowson Laboratory (2019) NatCen Social Research. National Diet and Nutrition Survey Years 1-9, 2008/2009-2016/2017, 15th ed. London: UK Data Service.

11. Blundell-Birtill P \& Hetherington MM (2019) Determinants of portion size in children and adolescents: insights from the UK National Diet and Nutrition Survey Rolling Programme 2008-2016. Nutrients 11, 2957.

12. Chawner LR, Blundell-Birtill P \& Hetherington MM (2020) Predictors of vegetable consumption in children and adolescents: analyses of the UK National Diet and Nutrition Survey (2008-2017). Br J Nutr 126(2), 295-306.

13. Toumpakari Z, Tilling K, Haase AM et al. (2019) High-risk environments for eating foods surplus to requirements: a multi level analysis of adolescents' non-core food intake the National Diet Nutrition Survey (NDNS). Public Health Nutr 22, 74-84.

14. Mak TN, Prynne CJ, Cole D et al. (2012) Assessing eating context and fruit and vegetable consumption in children: new methods using food diaries in the UK National Diet and Nutrition Survey Rolling Programme. Int J Behav Nutr Phys Act 9, 126

15. World Health Organisation (2003) Diet, Nutrition and the Prevention of Chronic Diseases. Report of a Joint WHO/ FAO Expert Consultation. Geneva: WHO.

16. Leahy KE, Birch LL, Fisher JO et al. (2008) Reductions in entree energy density increase children's vegetable intake and reduce energy intake. Obesity 16, 1559-1565.

17. Syrad H, Llewellyn CH, Johnson L et al. (2016) Meal size is a critical driver of weight gain in early childhood. Sci Rep $\mathbf{6}$, 28368.

18. Fisher JO \& Kral TVE (2008) Super-size me: portion size effects on young children's eating. Physiol Behav 94, $39-47$.

19. Porter A, Kipping R, Summerbell C et al. (2020) What guidance is there on portion size for feeding preschool-aged children ( 1 to 5 years) in the United Kingdom and Ireland? A systematic grey literature review. Obes Rev 21, e13021.

20. Bero LA, Norris SL \& Lawrence MA (2019) Making nutrition guidelines fit for purpose. BMJ 365, 11579.

21. Bates B, Collins D, Cox L et al. (2019) Years 1 to 9 of the Rolling Programme (2008/2009-2016/2017): Time Trend, Income Analyses. London: Gov.UK.

22. UK Data Service (2019) National Diet and Nutrition Survey Years 1-9, 2008/2009-2016/2017. https://beta.ukdata service.ac.uk/datacatalogue/studies/study?id=6533 (accessed May 2020).

23. Foster E, Hawkins A, Barton KL et al. (2017) Development of food photographs for use with children aged 18 months to 16 years: comparison against weighed food diaries - the Young Person's Food Atlas (UK). PLOS ONE 12, e0169084.

24. Fitt E, Cole D, Ziauddeen $\mathrm{N}$ et al. (2015) DINO (Diet In Nutrients Out) - an integrated dietary assessment system. Public Health Nutr 18, 234-241.

25. Murakami K \& Livingstone MB (2015) Variability in eating frequency in relation to adiposity measures and blood lipid 
profiles in British children and adolescents: findings from the National Diet and Nutrition Survey. Int J Obes 39, 608-613.

26. Piernas C \& Popkin BM (2011) Food portion patterns and trends among U.S. children and the relationship to total eating occasion size, 1977-2006. J Nutr 141, 1159-1164.

27. Taylor RW, Iosua E, Heath AM et al. (2017) Eating frequency in relation to BMI in very young children: a longitudinal analysis. Public Health Nutr 20, 1372-1379.

28. Toumpakari Z, Haase AM \& Johnson L (2016) Adolescents' non-core food intake: a description of what, where and with whom adolescents consume non-core foods. Public Health Nutr 19, 1645-1653.

29. World Health Organisation (2021) Body Mass Index-for-Age (BMI-for-Age). Geneva: WHO.

30. Cole TJ, Freeman JV \& Preece MA (1998) British 1990 growth reference centiles for weight, height, body mass index and head circumference fitted by maximum penalized likelihood. Stat Med 17, 407-429.

31. NatCen Social Research (2016) National Diet and Nutrition Survey Years 7 and 8 (2014/2015-2015/2016). List of Variables for UK Data. NDNS Years 7 to 8 Variable and Derived Variable Lists (ukdataservice.ac.uk) (accessed November 2021).

32. Office for National Statistics (2016) The National Statistics Socio-Economic Classification (NS-SEC): Office for National Statistics. https://www.ons.gov.uk/methodology/classifications andstandards/otherclassifications/thenationalstatisticssocio economicclassificationnssecrebasedonsoc2010 (accessed July 2020).

33. Rennie KL, Coward A \& Jebb SA (2007) Estimating underreporting of energy intake in dietary surveys using an individualised method. Br J Nutr 97, 1169-1176.

34. Torun B (2005) Energy requirements of children and adolescents. Public Health Nutr 8, 968-993.

35. Public Health England (2016) Eatwell Guide: Public Health England. https://assets.publishing.service.gov.uk/government/ uploads/system/uploads/attachment_data/file/528193/ Eatwell_guide_colour.pdf (accessed October 2021).

36. Rabe-Hesketh S \& Skrondal A (2012) Multilevel and Longitudinal Modelling Using Stata, 3rd ed. College Station, TX: Stata Press.

37. Huang TT, Howarth NC, Lin BH et al. (2004) Energy intake and meal portions: associations with BMI percentile in U.S. children. Obes Res 12, 1875-1885.

38. Burrows TL, Martin RJ \& Collins CE (2010) A systematic review of the validity of dietary assessment methods in children when compared with the method of doubly labeled water. J Am Diet Assoc 110, 1501-1510.

39. Tooze JA, Freedman LS, Carroll RJ et al. (2016) The impact of stratification by implausible energy reporting status on estimates of diet-health relationships. Biom J 58, 1538-1551.

40. Willett WC, Howe GR \& Kushi LH (1997) Adjustment for total energy intake in epidemiologic studies. Am J Clin Nutr 65, 1220S-1228S.

41. Vandenbroucke JP, von Elm E, Altman DG et al. (2007) Strengthening the Reporting of Observational Studies in Epidemiology (STROBE): explanation and elaboration. Ann Intern Med 147, W163-W194.

42. Fayet-Moore F, Peters V, McConnell A et al. (2017) Weekday snacking prevalence, frequency, and energy contribution have increased while foods consumed during snacking have shifted among Australian children and adolescents: 1995, 2007 and 2011-2012 National Nutrition Surveys. Nutr J 16, 65.

43. Wang JB, Patterson RE, Ang A et al. (2014) Timing of energy intake during the day is associated with the risk of obesity in adults. J Hum Nutr Diet 2, 255-262.

44. Duffey KJ, Pereira RA \& Popkin BM (2013) Prevalence and energy intake from snacking in Brazil: analysis of the first nationwide individual survey. Eur J Clin Nutr 67, 868-874.
45. Steenhuis I \& Poelman M (2017) Portion size: latest developments and interventions. Curr Obes Rep 6, 10-17.

46. Public Health England (2018) PHE Launches Change4Life Campaign around Children's Snacking. https://www.gov. uk/government/news/phe-launches-changeflife-campaignaround-childrens-snacking (accessed November 2021).

47. Infant \& Toddler Forum (2015) Portion Sizes for Toddlers. https://infantandtoddlerforum.org/toddlers-to-preschool/ portion-sizes-for-toddlers/toddler-portion-sizes-table/ (accessed October 2021).

48. Spill MK, Birch LL, Roe LS et al. (2010) Eating vegetables first: the use of portion size to increase vegetable intake in preschool children. Am J Clin Nutr 91, 1237-1243.

49. Patrick H \& Nicklas TA (2005) A review of family and social determinants of children's eating patterns and diet quality. J Am Coll Nutr 24, 83-92.

50. Welker EB, Jacquier EF, Catellier DJ et al. (2018) Room for improvement remains in food consumption patterns of young children aged 2-4 years. J Nutr 148, 1536S-1546S.

51. Robinson E, Jones A, Whitelock V et al. (2018) Over eating out at major UK restaurant chains: observational study of energy content of main meals. BMJ 363, k4982.

52. Meiselman HL (2008) Dimensions of the meal. J Foodservice 19, 13-21.

53. Larsen JK, Hermans RC, Sleddens EF et al. (2015) How parental dietary behavior and food parenting practices affect children's dietary behavior. Interact sources influence? Appetite 89, 246-257.

54. Johnson SL, Hughes SO, Cui X et al. (2014) Portion sizes for children are predicted by parental characteristics and the amounts parents serve themselves. Am J Clin Nutr 99, $763-770$.

55. Ruggiero CF, Hohman EE, Birch LL et al. (2021) INSIGHT responsive parenting intervention effects on child appetite and maternal feeding practices through age 3 years. Appetite 159, 105060.

56. Daniels LA, Magarey A, Battistutta D et al. (2009) The NOURISH randomised control trial: positive feeding practices and food preferences in early childhood - a primary prevention program for childhood obesity. BMC Public Health 9, 387.

57. Reeves S, Wake Y \& Zick A (2011) Nutrition labeling and portion size information on children's menus in fast-food and table-service chain restaurants in London, UK. J Nutr Educ Behav 43, 543-547.

58. Andrade GC, Gombi-Vaca MF, Louzada M et al. (2020) The consumption of ultra-processed foods according to eating out occasions. Public Health Nutr 23, 1041-1048.

59. Wansink B \& van Ittersum K (2007) Portion size me: downsizing our consumption norms. J Am Diet Assoc 107, 1103-1106.

60. HM Government (2016) Childhood Obesity: A Plan for Action - GOV.UK (www.gov.uk) (accessed October 2021).

61. Foster E \& Bradley J (2018) Methodological considerations and future insights for 24-hour dietary recall assessment in children. Nutr Res 51, 1-11.

62. Colapinto CK, Fitzgerald A, Taper LJ et al. (2007) Children's preference for large portions: prevalence, determinants, and consequences. J Am Diet Assoc 107, 1183-1190.

63. Reale S, Simpson RM, Marr C et al. (2019) Snack portion sizes for preschool children are predicted by caregiver portion size, caregiver feeding practices and children's eating traits. Nutrients 11, 3020.

64. Kling SM, Roe LS, Keller KL et al. (2016) Double trouble: portion size and energy density combine to increase preschool children's lunch intake. Physiol Behav 162, 18-26.

65. Orlet Fisher J, Rolls BJ \& Birch LL (2003) Children's bite size and intake of an entree are greater with large portions than with age-appropriate or self-selected portions. Am J Clin Nutr 77, 1164-1170. 
66. Fisher JO (2007) Effects of age on children's intake of large and self-selected food portions. Obesity 15, 403-412.

67. Looney SM \& Raynor HA (2011) Impact of portion size and energy density on snack intake in preschool-aged children. $J$ Am Diet Assoc 111, 414-418.

68. McCrickerd K, Leong C \& Forde CG (2017) Preschool children's sensitivity to teacher-served portion size is linked to age related differences in leftovers. Appetite 114, 320-328.

69. Mooreville M, Davey A, Orloski A et al. (2015) Individual differences in susceptibility to large portion sizes among obese and normal-weight children. Obesity 23, 808-814.

70. Hall KD, Heymsfield SB, Kemnitz JW et al. (2012) Energy balance and its components: implications for body weight regulation. Am J Clin Nutr 95, 989-994.

71. Hebestreit A, Bornhorst C, Barba G et al. (2014) Associations between energy intake, daily food intake and energy density of foods and BMI z-score in 2-9-year-old European children. Eur J Nutr 53, 673-681.

72. ESPGHAN Committee on Nutrition, Agostoni C, Braegger C et al. (2011) Role of dietary factors and food habits in the development of childhood obesity: a commentary by the
ESPGHAN Committee on Nutrition. J Pediatr Gastroenterol Nutr 52, 662-669.

73. Fayet $F(2012)$ Energy distribution patterns in Australia and its relationship to age, gender and body mass index among children and adults. Nutr Diet 69, 102-110.

74. Burrows T, Goldman S \& Rollo M (2020) A systematic review of the validity of dietary assessment methods in children when compared with the method of doubly labelled water. Eur J Clin Nutr 74, 669-681.

75. DiSantis KI, Birch LL, Davey A et al. (2013) Plate size and children's appetite: effects of larger dishware on self-served portions and intake. Pediatrics 131, e1451-e1458.

76. Robinson E, Nolan S, Tudur-Smith C et al. (2014) Will smaller plates lead to smaller waists? A systematic review and meta-analysis of the effect that experimental manipulation of dishware size has on energy consumption. Obes Rev 15, 812-821.

77. Burki TK (2016) Sugar tax in the UK. Lancet Oncol 17, e182.

78. Steenhuis IH \& Vermeer WM (2009) Portion size: review and framework for interventions. Int J Behav Nutr Phys Act 6, 58 . 